\title{
The nature of the 'knee' in the cosmic ray energy spectrum
}

\author{
A.D.Erlykin ${ }^{\mathrm{a}, \mathrm{b}}$ and A.W.Wolfendale ${ }^{\mathrm{b}}$ \\ (a) P.N.Lebedev Physical Institute, Leninsky prosp. 53, Moscow 119991, Russia \\ (b) Department of Physics, University of Durham, South Road, Durham DH1 3LE, UK \\ Presenter: A.W.Wolfendale(a.w.wolfendale@durham.ac.uk), uki-wolfendale-AW-abs1-og12-oral
}

Attention is directed again at two aspects of the well-known 'knee' in the cosmic ray energy spectrum at 3PeV: the mass of the predominant particles at this energy and their source, in view of recent developments. Earlier, we had used a particular acceleration model [1] to infer that the particles are mainly oxygen nuclei [2]; direct measurements, when extrapolated [3], gave a similar result. Initially, [2] no specific source was identified. More recently, however, [4] we have specified the Monogem Ring supernova remnant [5] as the likely source; this is at just the right distance and age and the energies are reasonable.

Concerning the mass composition at the knee, recent direct measurements, [6], show a likely flattening in the spectrum above $\sim 10^{4} \mathrm{GeV} /$ nucleon for He-nuclei, a flattening which, if extrapolated to higher energies, would meet the measured spectrum in the knee region. He-nuclei in the knee region would also be marginally more consistent with KASCADE extensive air shower data [7].

\section{Introduction}

A variety of features has led to uncertainties in cosmic ray origin, even at PeV energies (the 'knee region' ). In our own model $[2,4,8]$, the knee is largely populated by oxygen nuclei from a single recent, local source ( or CNO ). The most comprehensive indirect study has been made by the KASCADE EAS array ( [7] and see [9] ) but there is ambiguity in the mass assignments due to uncertainty with the model for the high energy physics involved in the analysis. The identification of the predominant mass in the knee region is equivocal: $\mathrm{He}$ or $\mathrm{CNO}$ ( i.e. there is no unambiguous support for our $\mathrm{CNO}$ - identification ).

\section{Indirect evidence favoring predominant He at the knee}

There are several indirect indications for the predominance of helium at the knee rather than oxygen ( or $\mathrm{CNO})$ :

(i) In the series of recent models of particle acceleration in SNR the maximum rigidity is essentially higher than the 0.4 PV adopted by us from [1] and used as an argument for the knee position at $\sim 3-4 \mathrm{PeV}$ to be attributed to predominant oxygen $\left(\mathrm{Z}=8\right.$ ). For example in [10] $\mathrm{R}_{\max }=15 \mathrm{PV}$. There is thus the possibility of attributing the position of the knee to a lighter element than oxygen.

(ii) In [3] we analysed the possibility of different models of CR interaction giving a consistent result for the primary mass composition, measured by different combinations of data. We concluded that the best consistency is for the QGSJET model - and here the dominant element at the knee derived from the $N_{\mu}-N_{e}$ distribution obtained by KASCADE with the help of the QGSJET model, is He.

(iii) In [9] we stressed the fact that according to the latest analysis of KASCADE data the mass composition changes very rapidly beyond the knee, becoming heavier. We have compared two versions of the Single Source model of the knee: with $\mathrm{O}$ or He dominant at the knee with these data. The results show that the dominance of $\mathrm{He}$, its sharp cutoff and transition to dominant $\mathrm{O}$ in the region of the second peak $(\sim 12-16$ $\mathrm{PeV}$ ), gives a much faster rise of $\langle\ln A\rangle$ and fits the experimental data better. 
(iv) The analysis of the hadron energy $E_{h}$ in EAS detected by an ionization calorimeter at mountain level

[11] showed the existence of remarkable peaks and troughs in the mean ratio $\left\langle E_{h} / N_{e}\right\rangle$. Their position, separation and excursion from the smooth dependence ( positive or negative ) analysed on the basis of simulations [12], which give $\mathrm{Ne}\left(\mathrm{E}_{0}, \mathrm{~A}\right)$ as a function of the energy $\mathrm{E}_{0}$ and the mass $\mathrm{A}$ of the primary $\mathrm{CR}$ nucleus for the Tien-Shan observation level ( $3340 \mathrm{~m}$ a.s.l. ) show better agreement with the predominance of He in the knee ( Figure 1 ).

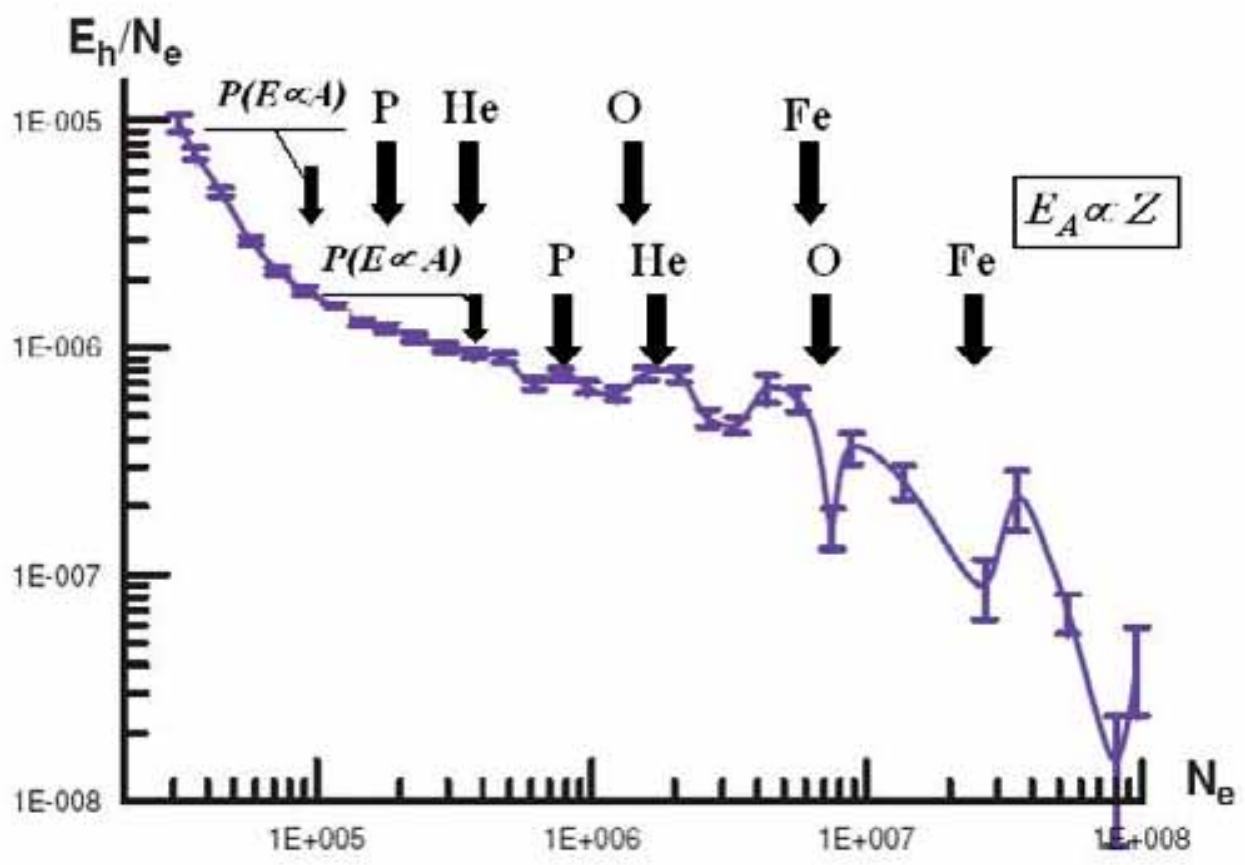

Figure 1. The energy of the EAS hadron component $E_{h}$, referred to the EAS size $N_{e}$, as a function of $N_{e}$ for EAS observed at the mountain level [11]. The upper line of arrows indicates positions of energy cutoffs expected for the SSM with predominant oxygen $(\mathrm{O})$ at the knee ( the knee at Tien-Shan level is observed at $\log \mathrm{N}_{\mathrm{e}} \approx 6.15$ ). The lower line of arrows indicates these positions for helium (He) predominant at the knee. The rigidity cutoff for the upper identification is $0.4 \mathrm{PV}$ and that for the lower, $1.5 \mathrm{PV}$. The position of the left arrow at both lines denoted as $P(E \infty A)$ corresponds to the position of the cutoff for protons if energy cutoffs $\mathrm{E}_{\mathrm{A}}$ are proportional to A instead of $Z$. In this case, the relative positions of the other nuclear groups would barely change.

(v) Although it is too early to make a firm conclusion, due to unsufficient statistics, in the analysis of the mass composition made by KASCADE there is a hint for the existence of a 'third' peak of intensity [7]. The points at 50-70 PeV have a positive excursion from the smooth behaviour both for the QGSJET and SYBILL models, due presumably to Fe. The errors are still large but one has to keep this irregularity in mind and wait for the data from KASCADE-GRANDE. If the existence of this 'third' peak at 50-70 PeV is confirmed, then taking into account the possibility of a small overestimation of the primary energy for cascades induced by the heavy nuclei such as Fe [13], it can provide strong evidence that the first peak at 3-4 PeV, ie. the knee, is due to the dominance of He. 
(vi) More important is the publication of new results from a direct measurement by'ATIC' [6]. The data go up to $\sim \log \mathrm{E}_{\mathrm{n}}=5.0$ for protons, 4.3 for He-nuclei ( $\mathrm{E}_{\mathrm{n}}$ being the energy per nucleon) and 3.5 for $\mathrm{CNO}$ and $\mathrm{Fe}$. Interestingly, there is some evidence for an upturn in the helium spectrum starting at about $\log \mathrm{E}_{\mathrm{n}}=4.0$ such that if it is permitted to extrapolate by a factor 30 to reach the knee energy the necessary intensity is achieved. For CNO nuclei, where we drew attention to the flattening of the spectrum starting from $\log \mathrm{E}_{\mathrm{n}}=2$ ( from the JACEE data [14] of reference [3] ), there is a smaller indication in the ATIC data. The situation is thus that the emphasis from direct measurements has moved somewhat from a CNO-knee to a He-knee; it is, however, premature to be certain.

All these arguments taken separately are not overwhelming - just indications, but the total number of them is already large and growing with time, so that one has to take this 'collection of indications' seriously.

\section{The Monogem Ring SNR}

In a previous paper [4] we made the case for the Monogem Ring SNR being 'at the right distance and being of the right age' to satisfy our single source model. The calculations [4] showed agreement with the measured intensity, expressed in terms of rigidity, at the knee, assuming CNO for the component which gives the predominant contribution at the knee. Since then we have found that the rigidity spectrum of CR expected from our Single Source had been overestimated. The true rigidity spectrum of particles expected for our Single Source is lower in intensity and agreement between the model with oxygen predominant at the knee is damaged.

Reworking for helium, and allowing for the fact that the energy released in the SN was only $1.9 \times 10^{51} \mathrm{erg}$, we get good agreement, however! The situation is indicated in Figure 2. At this stage a comment should be made about the clumpiness of the Monogem Ring - a feature shown by virtually all SNR. Models predict a relationship between SN energy released, the subsequent CR output and the maximum energy attainable. It seems that one should decouple the latter two: the CR energy content will depend on the energy released, but the maximum particle energy will depend on the energy density in the 'knots'. Thus, it will be possible to achieve a low CR intensity but a high $\mathrm{E}_{\max }$.

\section{Conclusion}

New evidence gives rather more credence to the suggestion that helium rather than oxygen nuclei are responsible for the knee. The evidence comes from hints from new direct spectral measurements and several indirect indications, both experimental and theoretical. The evidence is not proof, but the growing number of indications allows one to consider this suggestion as a more likely option. Concerning our identification of the knee as indicating a significant contribution from a local, single, recent SN, our view is unchanged.

\section{References}

[1] Berezhko, E G et al, 1996 JETP. 82, 1

[2] Erlykin, A D and Wolfendale, A W, 1997, J.Phys.G. 23, 979

[3] Erlykin, A D et al, 1998, Astropart.Phys. 8, 282

[4] Erlykin, A D and Wolfendale, A W, 2003, J.Phys.G, 29, 709

[5] Thorsett, S E et al, 2003, Ap.J.Lett., 592, L71

[6] Zatsepin, V I et al, 2004, Izv.RAN, ser.fiz. 68, 1593 and this conference.

[7] Kampert, K H , 2004, Nucl.Phys.B (Proc.Suppl.), 136, 273

[8] Erlykin, A D and Wolfendale A W, 2001, J.Phys.G. 27, 1005

[9] Erlykin, A D and Wolfendale A W, 2005, Astropart.Phys., 23, 1[10] Bell, A R and Lucek, S G, 2001, Mon.Not.Roy.Astr.Soc., 321, 433 
[11] Barnaveli, T T et al., 2004, $28^{\text {th }}$ RCRC, Moscow ( in Russian - to be published in Izv.RAN, ser.fiz ), astro-ph/0310524

[12] Danilova, T V et al., 1993, J.Phys.G, 19, 429

[13] Erlykin, A D and Wolfendale, A W, 2004, Nucl.Phys.B (Proc.Suppl.), 136, 282 ; J.Phys.G: Nucl.Part.Phys., 2005, 31, 791

[14] Shibata, T, 1995, $24^{\text {th }}$ ICRC, Rome, Inv.Rapp.High Papers, 713

[15] Erlykin, A D and Wolfendale, A W, 2005 ( in preparation )
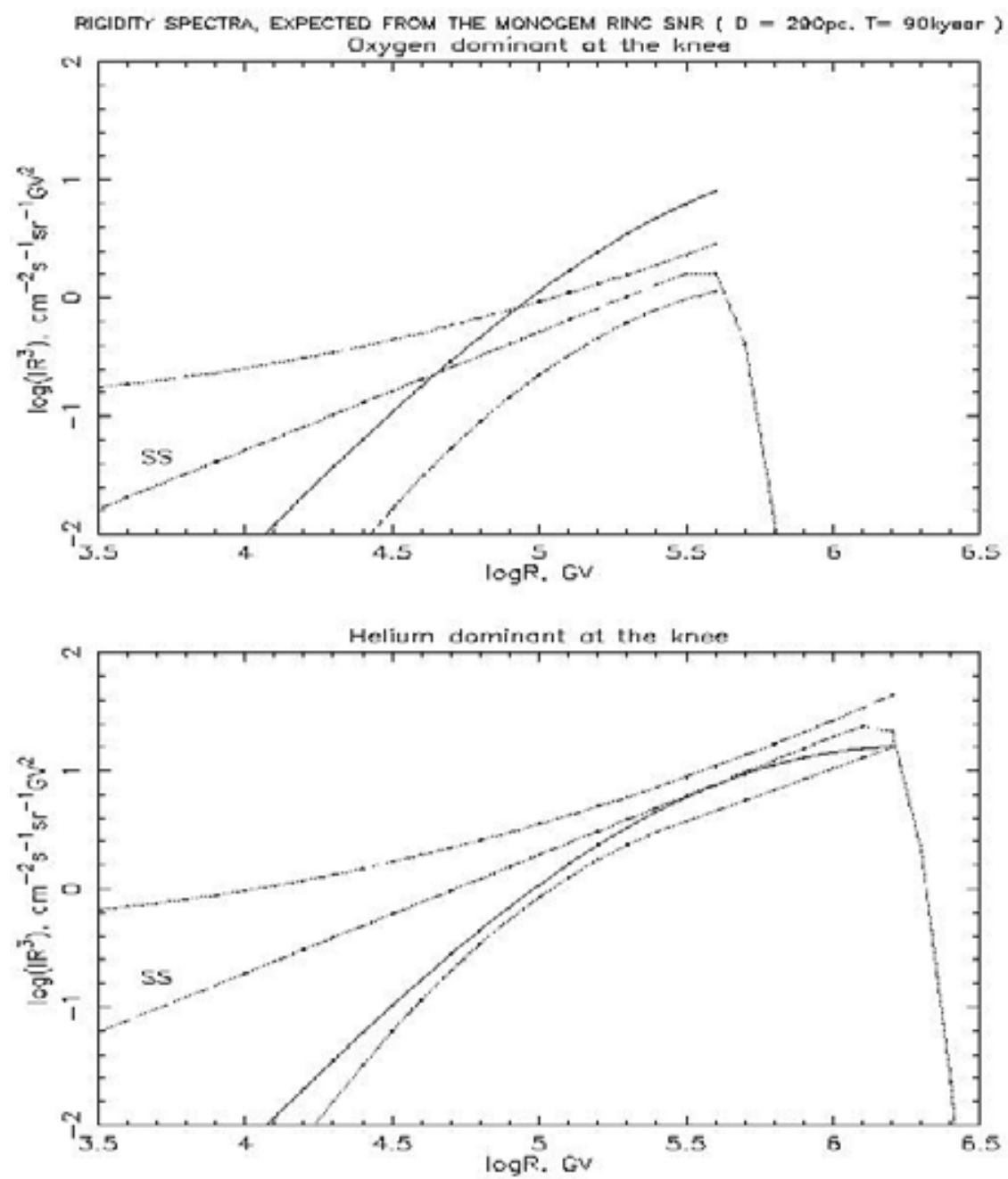

Figure 2. Comparison of the rigidity spectrum of particles expected from the Monogem Ring SNR with a distance of $290 \mathrm{pc}$, age - of 90 kyear, an explosion energy of $1.9 \times 10^{50} \mathrm{erg}$ and that derived from the Single Source model assuming oxygen ( upper panel ) or helium ( lower panel ) predominant at the knee. There is clearly a consistent picture for the knee being due, principally, to helium nuclei being accelerated by the Monogem Ring SNR at the currently predicted distance and age [5]. 'SS' and the accompanying converging upper and lower lines represent our needed single source spectrum and its intensity range. 